of microchemical 'factories' on a chip. Wetted fibers will require easier ways of inserting and removing the fibers from the chip as well as simpler methods of preparing the fiber tips with biomolecules for detection.

An LOC system must have a number of generic components: a method of introducing reagents and samples; methods for moving these fluids around on the chip, and for combining and mixing them; and various other items such as detectors for most microanalytical work, as well as components for purification of products for systems used in synthesis. The field has, so far, centered on demonstrating concepts for these components. In the future there will be sufficient methods of fabrication, and a range of components which will make it possible to apply microfluidic systems to the resolution of problems rather than simply to the demonstration of principles [3].

\section{Cross References}

- Flow Cytometer Lab-on-Chip Devices

- Spectrophotometric Analysis

- Capillary Electrochromatography

- Spectrofluorometry

- Immunoassay

- Capillary Electrophoresis

\section{References}

1. Bailey D, Wright E (2003) Practical Fiber Optics. Elsevier, Burlington

2. Shotwell RA (ed) (1997) An Introduction to Fiber Optics. Prentice Hall, Englewood Cliffs

3. Whitesides GM (2006) The origins and the future of microfluidics. Nature 442(27):368-373

4. Grattan KTV, Sun T (2000) Fiber Optic Sensor Technology: Introduction. In: Grattan KTV, Meggitt BT (eds) (2000) Optical Fiber Sensor Technology. Kluwer Academic, Dordrecht

5. Zhang ZY, Grattan KTV (1998) Commercial activity in optical fiber sensors. In: Grattan KTV, Meggitt BT (eds) (1998) Optical Fiber Sensor Technology. Kluwer Academic, Dordrecht, pp 257-306

6. Lin CH, Lee GB, Chen SH, Chang GL (2003) Micro capillary electrophoresis chips integrated with buried SU-8/SOG optical waveguides for bio-analytical applications. Sens Actuators A 107:125-131

7. Bowden M, Song L, Walt DR (2005) Development of a microfluidic plattform with an optical imaging microarray capable of attomolar target DNA detection. Anal Chem 77:5583-5588

8. Treflak NR, Barnes J, Rask F, Courtney DG, Walford R, Li R, Oleschuk RD, Loock HP (2005) Absorption measurements in microfluidic devices using ring-down spectroscopy. Proc SPIE 5969:596900

9. Camou S, Fujita H, Fujii T (2003) PDMS 2D optical lens integrated with microfluidic channels: principle and characterization. Lab Chip 3:40-45

10. Golonka LJ, Zawada T, Radojewski J, Roguszczak H, Stefanow M (2006) LTCC microfluidic system. Int J Appl Ceram Technol 3:150-156
11. Golonka LJ, Roguszczak H, Zawada T, Radojewski J, Grabowska I, Chudy M, Dybko A, Brzozka Z, Stadnik D (2005) LTCC based microfluidic system with optical detection. Sens Actuators B 111-112:396-402

12. Wang Z, El-Ali J, Engelund M, Gotsaed T, Perch-Nielsen IR, Morgensen KB, Snakenborg D, Kutter JP, Wolf A (2004) Measurements of scattered light on a microchip flow cytometer with integrated polymer based optical elements. Lab Chip 4:372-377

13. Qi S, Liu X, Ford S, Barrows J, Thomas G, Kelly K, McCandless A, Lian K, Goettert J, Soper SA (2002) Microfluidic devices fabricated in poly(methylmethacrylate) using hot-embossing with integrated sampling capillary and fiber optics for flourescence detection. Lab Chip 2:88-95

14. Chabinyc ML, Chiu DT, McDonald JC, Stroock AD, Christian JF, Karger AM, Whitesides GM (2001) An integrated fluorescence detection system in poly(dimethylsiloxane) for microfluidic applications. Anal Chem 73:4491-4498

15. Lucas LJ, Han JH, Chesler N, Yoon JY (2007) Latex immunoagglutination assay for a vasculitis marker in a microfluidic device using static light scattering detection. Biosens Bioelectron 22:2216-2222

16. Hsieh HB, Marrinucci D, Bethel K, Curry DN, Humphrey M, Krivacic RT, Kroener J, Kroener L, Ladanyi A, Lazarus N, Kuhn P, Bruce RH, Nieva J (2006) High speed detection of circulating tumor cells. Biosens Bioelectron 21:1893-1899

\section{On-Chip Electrospray}

LESLIE YeO, JAMES FRIEND

Micro/Nanophysics Research Laboratory

Department of Mechanical Engineering, Monash

University, Clayton, VIC, Australia

leslie.yeo@eng.monash.edu.au

\section{Synonyms}

Electrospray ionization-mass spectrometry (ESI-MS); Electrospray mass spectrometry; Electrospray emitter onchip; Chip electrospray mass spectrometry

\section{Definition}

Electrospraying utilizes an applied electric field to generate micron or nanometer sized aerosol drops. If DC fields are employed, the emitted drops carry a net charge which allows electrosprays to function as ionization sources, in particular, for mass spectrometry. With electrospray ionization (ESI), interfacing between microfluidic separation technology such as on-chip high performance liquid chromatography (HPLC) and capillary electrochromatography (CEC) with mass spectrometry (MS) is now realizable.

\section{Overview}

The concept of employing electrostatic charging to atomize a liquid source was first observed by John Zeleny in 


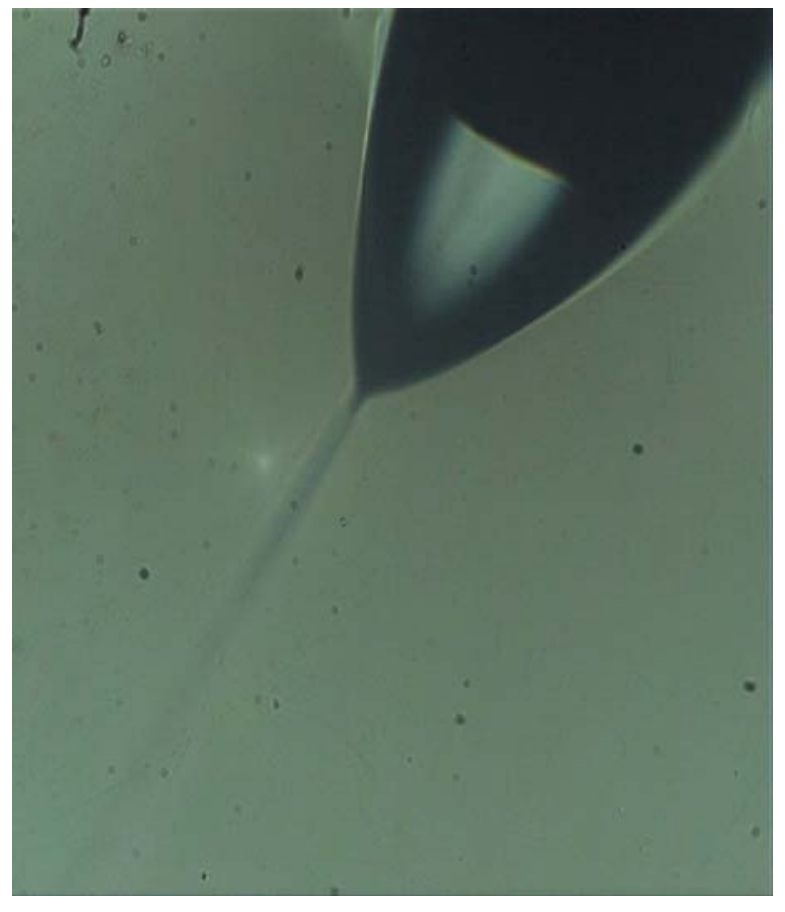

On-Chip Electrospray, Figure 1 Taylor cone formation at the tip of a $100 \mu \mathrm{m}$ capillary across which a $3 \mathrm{kV}$ DC voltage is applied. A slender jet issues from the tip of the Taylor cone as a result of Rayleigh fission, subsequently breaking up to form aerosol droplets

1914. If the liquid is flowing through a metal capillary, the surface of which is raised to a potential with respect to a grounded source placed a small distance away, the electric field acts to stretch and deform the liquid meniscus at the capillary tip. The fluid physics under the influence of an electric field in such circumstances was described by Sir Geoffrey Ingram Taylor in 1964, who observed the meniscus to deform into a sharp cone with a specific half angle of $49.3^{\circ}$ for highly conducting liquids. This Taylor cone, depicted in Fig. 1, arises due to the separation of electrical charges within the liquid or ambient phase to form a thin polarized layer of counter-ions near the meniscus interface; these counter-ions then drift towards the tip of the meniscus by tangential electromigration.

The counter-ion accumulation at the tip then creates a Coulombic repulsion force which causes the tip to disintegrate into a thin jet that emanates from meniscus when the surface tension force holding the drop together is exceeded, as shown in Fig. 1. This jet subsequently breaks up due to hydrodynamic or Coulombic instabilities to form a spray of small liquid drops. As the accumulated charge at the tip is transferred from the meniscus tip to the jet and subsequently to the drops, each individual drop carries a net charge. Since the size of the ejected drop shrinks during its flight due to evaporation, the repulsive force builds up due to the increase in the charge density. Coulombic fission then occurs once again when the Rayleigh limit

$$
q>64 \pi^{2} \varepsilon_{\mathrm{m}} a^{3} \gamma,
$$

is reached. In Eq. (1), $q$ is the charge, $\varepsilon_{\mathrm{m}}$ the permittivity of the surrounding medium, $a$ the drop diameter and $\gamma$ the surface tension. The drop disintegrates to produce even smaller droplets, which themselves undergo similar disintegration cycles, as depicted in Fig. 2; this Rayleigh instability fission cascade eventually leads to charged drops with dimensions down to $10 \mathrm{~nm}$ orders.

The ability of the electrospray to produce very small, monodispersed charged drops then naturally lends itself as a powerful soft ionization technique for the mass spectrometry of large, complex and non-volatile biomacromolecules (e. g., DNA, peptides and proteins) which are otherwise denatured by other ionization techniques. Electrospray ionization (ESI) also has several advantages over matrix-assisted laser desorption/ionization (MALDI), which is another soft ionization technique. The use of lasers requires fine tuning of optical properties in order to ensure quality and reproducibility in the results; signals are dependent on the laser wavelength, pulse energy, time between pulses and impact angle, amongst others. Moreover, the inorganic salts in proteins can interfere with the ionization and hence elaborate sample preparation to remove these components is a vital step in MALDI. The small size of the laser spot compared to the sample also requires multiple laser pulses targeted at different sample regions in order to obtain a statistical average of the local concentration within the sample, which is further complicated by inhomogeneity due to partitioning of the analyte molecules as a result of non-uniform solvent evaporation. Sample dispensing onto MALDI target plates also requires the integration of complex components such as piezoelectric microdispensers or centrifugal delivery systems.

ESI was first proposed by Malcolm Dole in 1968 who noticed that the Coulombic fission cascade would eventually lead to sufficiently small drops which contained a single solute molecule that retained some of the drop charge such that a fully desolvated gas phase ion would ultimately be left once all the solvent evaporates, this mechanism being known as the charge residue model (CRM). These efforts were, however, largely unsuccessful practically due to the use of an ion-drift spectrometer to which the electrospray was interfaced. It was John Fenn, then at Yale University, who later developed a practical method for electrospray ionization mass spectrometry (ESI-MS) that allowed the identification and structure analysis of biomacromolecules of virtually unlimited molecular weights to an accuracy of $0.01 \%$ by averaging 


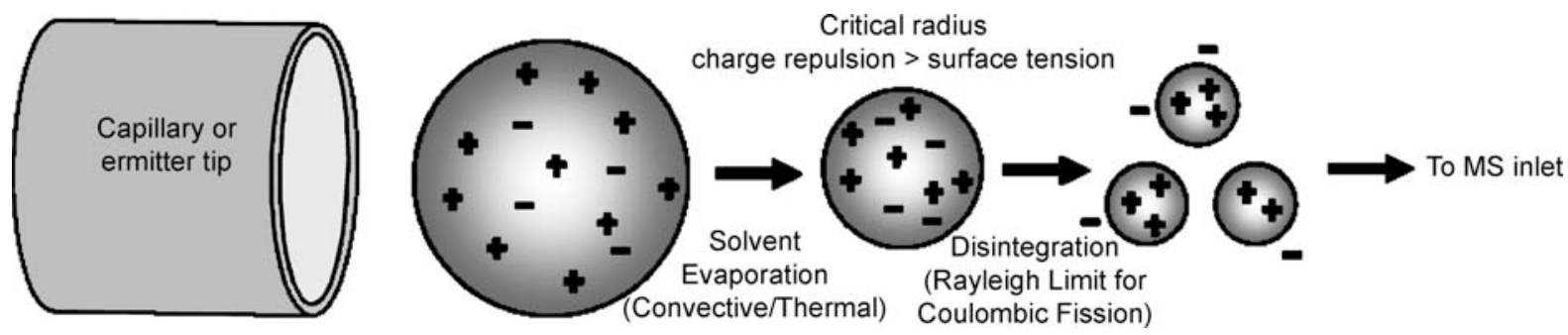

On-Chip Electrospray, Figure 2 Schematic illustration of the Coulombic fission process occurring during DC electrospraying giving rise to successively smaller charged drops

over the signals generated by the multiple ions which are associated with the high mass-to-charge ratios of large, complex molecules [1]. In recognition of this significant contribution and its impact on genomic, proteomic and drug discovery research, Fenn was awarded the Nobel Prize in Chemistry in 2002. It should however be noted that the term electrospray ionization is somewhat misleading as the process is not akin to electron ionization in which electroneutral molecules are converted into ions. Instead, the electrospray essentially allows the transfer of analytes, already ionized in the liquid phase, into the gas phase [2]. Nevertheless, we will retain the term due to its widespread use in the literature.

The recent progress in micro/nanofabrication technology as well as microfluidics has spurred efforts to interface chip-based separation devices with electrospray ionization mass spectrometers. As frontiers in the ability to miniaturize the mass analyzers are being extended further, an integrated field portable microdevice that couples both on-chip separation (e.g., capillary electrophoresis, capillary electrochromatography and high-performance liquid chromatography) and analytical technology with an electrospray interface to produce a complete tool, which is both reliable and can be easily replicated, is increasingly realizable. Such a device would be extremely attractive for in-situ analysis in environmental, chemical or biological monitoring and remediation, offering the advantage of reduced analyte quantities and separation times, higher flow rate capabilities, low cost, increased precision and repeatability. At the time of writing, an ESI-MS microdevice for high performance liquid chromatography known as the HPLC-chip which is marketed by Agilent Technologies is commercially available. The HPLC-chip integrates the HPLC column with a sample enrichment column and an electrospray tip, which can then be mounted onto a mass spectrometer.

\section{Basic Methodology}

A review of DC electrospraying is given by Grace \& Marijnissen [3]. It should be noted that the use of high fre- quency $(>10 \mathrm{kHz}) \mathrm{AC}$ electric fields gives rise to completely different drop dynamics and characteristics [4], the most important distinction perhaps being the larger $1-10 \mu \mathrm{m}$ drops generated which are electroneutral. For the purposes of on-chip electrospray devices for analyte separation and detection, we will focus on the use of DC electrospraying henceforth as the AC electrospray has only recently been discovered and hence the use of $\mathrm{AC}$ fields for various applications and devices is currently work-inprogress (see, for example, [5]).

The most stable DC electrospray mode required for ionization is the cone-jet mode [3] described above and depicted in Fig. 1. The appearance of this characteristic conical meniscus and its stability has therefore been generally, and more likely than not, universally, adopted as the benchmark criterion for stable electrospray ionization. This stability is most commonly disrupted intermittently by gas bubbles aggregating into large slugs, thus temporarily causing cessation in the spraying. Once the slug is released, the spraying resumes. The formation of gas bubbles is a common problem in DC electrokinetics as a result of electrolytic reactions occurring to balance the charge induced by the applied voltage at the metal capillary, leading to a reduction in throughput and hence stable electrospray disruption due to the enormous capillary pressures. Joule heating and the generation of ionic contaminant as a result of Faradaic reactions at the electrodes are also additional problems accompanying the use of large DC voltages. Alternatively, stable electrospraying can also be disrupted by stray electric fields, mechanical perturbations (e. g., vibration) or flow variation.

The critical voltage $V_{\mathrm{c}}$ required for stable electrospraying can be estimated through a balance between the capillary stress, of order $\gamma / R$, where $R \sim 10^{-3}-10^{-4} \mathrm{~m}$ is the principal curvature of the meniscus, of the same order as the radial capillary dimension, and the electric stress $\varepsilon_{1} V^{2} / d^{2}$, where $\varepsilon_{1}$ is the liquid permittivity, $V$ the applied voltage and $d$ the electrode separation:

$$
\frac{V_{\mathrm{c}}}{d} \sim \sqrt{\frac{\gamma}{\varepsilon_{1} R}} .
$$


Typically, $\quad \gamma \sim 10^{-2} \mathrm{~kg} / \mathrm{s}^{2}, \quad d \sim 10^{-2} \mathrm{~m} \quad$ and $\quad \varepsilon_{1} \sim$ $10^{-10} \mathrm{C}^{2} /(\mathrm{Jm})$, thus suggesting that extremely large critical fields in excess of $1 \mathrm{kV}$ are required.

Taylor, by considering a static balance between capillary and electric stresses, showed the existence of a conical meniscus with a half angle of $49.3^{\circ}$ at equilibrium for a perfectly conducting liquid. In this limit, the meniscus behaves as a constant potential body and hence the field is external to the drop; the field lines intersect the interface orthogonally. The normal gas phase electric field $E_{\mathrm{n}}$ then scales as $1 / \sqrt{R}$, and hence the normal electric stress $p_{\mathrm{E}} \sim E_{\mathrm{n}}^{2}$ scales as $1 / R$. At every point $R$ along the interface, the normal electric stress exactly balances the azimuthal capillary pressure $p_{\mathrm{C}} \sim \gamma / R$. This exact balance and absence of a specific length scale, is responsible for the formation of a static Taylor cone [6].

The perfectly conducting limit was later relaxed to allow for the effect of finite liquid conductivity [7]. In these cases in which the liquid meniscus is assumed to be sufficiently slender such that the longwave approximation can be assumed, the field that arises is predominantly in the liquid phase, tangential to the interface. Nevertheless, this tangential liquid phase electric field $E_{\mathrm{t}}$ also scales as $1 / \sqrt{R}$ and thus an exact balance between the normal electric stress $p_{\mathrm{E}} \sim E_{\mathrm{t}}^{2}$ and the capillary stress $p_{\mathrm{C}} \sim \gamma / R$ again yields a stable equilibrium cone-like meniscus. The cone angle, however, depends crucially on the liquid to gas permittivity ratio $\varepsilon_{1} / \varepsilon_{\mathrm{m}}$, wherein the Taylor angle is recovered in the perfectly conducting limit as $\varepsilon_{1} / \varepsilon_{\mathrm{m}} \rightarrow \infty$ [6].

An alternative ionization mechanism to Dole's charge residue model (CRM) above has been proposed, in part to account for the inadequacy of CRM to account for the excess of analyte molecules over the observed charges in the electrospray flux. The CRM predicts that the relative number of drops for each species is proportional to the species relative concentration. The difficulty lies conceptually in the unlikely possibility for the successive drop subdivisions during the disintegration process to end up with the observation that all the daughter drops contained only a single analyte molecule for each of the ionizable species, especially in the presence of strong evaporation currents. Even if this were possible, a spectral peak for each species would always be obtained, which is not true. The alternative model, in contrast, proposes that the singly charged or multiply charged gas phase ions are generated by the evaporation of small, highly charged liquid drops of the analyte species [8].

\section{On-Chip ESI-MS Interfacing}

Progress in the development of on-chip electrospray interfaces for mass spectrometry has grown exponentially over the past two decades. Whilst there are many different designs and methods proposed, it is possible to summarize these developments in general into three broad categories based on the nature of the electrospray emitter type employed: Blunt edge, transfer capillary attachment, and, emitter tip. Early developmental work employed the blunt edge configuration. However, there appeared to be a subsequent parallel divergence thereafter, in which either the transfer capillary or the emitter tip interfaces were employed. Most of the recent on-chip electrospray interface designs therefore fall generally into either of these two latter categories.

On-chip ESI-MS devices were initially fabricated onto quartz or silica substrates. In addition to problems due to the hydrophobicity (discussed below), fabrication on glass is relatively labor-intensive. The shapes and dimensions available for patterning on glass are also limited. Silicon has also been commonly used because of its amenability to fabricating relatively small high aspect ratio features such as needle tip structures. In addition, semiconductor processing techniques such as deep reactive ion etching (DRIE) can be carried out for mass production of silicon chips [9]. However, silicon being semi-conducting has the disadvantage of current leakage.

Recently, the use of polymer-based substrates such as parylene, poly(dimethylsiloxane) or PDMS, polycarbonate (PC), polyimide (PI), poly(methylmethacrylate) or PMMA, poly(ethylene terephthalate) or PET, amongst others, has become increasingly attractive due to the availability of a wide range of plastics at low costs and the relatively simple and rapid microfabrication (rapid prototyping) techniques involved. The flexibility, superior optical properties and the ability to bond readily to glass after exposure to plasma of some plastics also make these materials attractive. Plastics, and glass for that matter, however, often have to be treated with hydrophobic surfactants to eliminate wetting and capillary ballooning at emitter tips. Other methods to avoid emitter tip wetting are discussed in the next section.

Electrospray tips from SU-8 photoepoxy have also been fabricated. Nevertheless, solvent resistance of polymerbased substrates is a critical issue, with poor resistance resulting in the swelling and distortion of the channels. A comparison between the performance of fused silica, parylene and borosilicate glass is discussed in Licklider et al. [10].

\section{Blunt Edge Orifice}

The first miniaturized on-chip electrosprays consisted of a planar glass microchip with $60 \mu \mathrm{m}$ wide and $10 \mu \mathrm{m}$ deep etched channels [11]. The open-end channel exit 


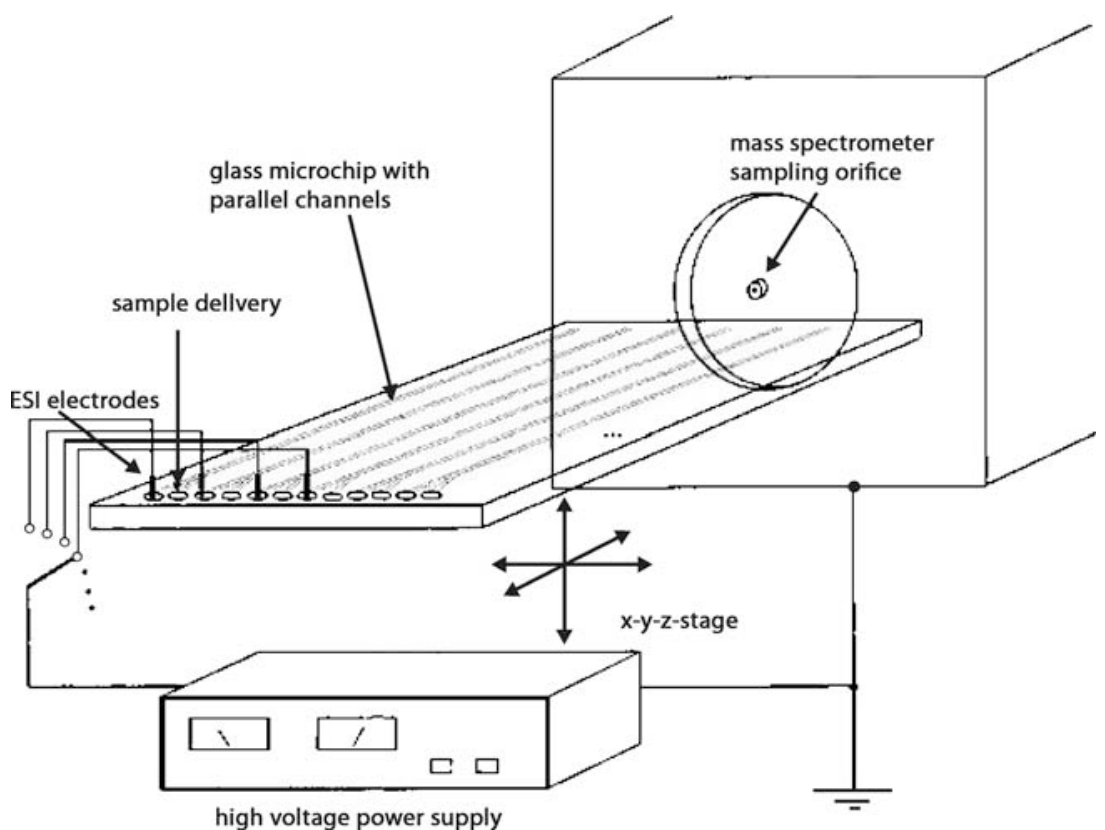

On-Chip Electrospray, Figure 3 Schematic diagram representing the multichannel ESI-MS chip device in Xue et al. [12]. Each channel was connected to two reservoirs for sample injection and delivery. A syringe pump attached to the second reservoir of each channel was used to deliver the sample through the channels and electrodes were placed in one of each two buffer reservoirs to provide the electrical contact with the solution. The electrospray orifices consisted of the blunt edges of the open-ended channels. Reprinted with permission from [12]

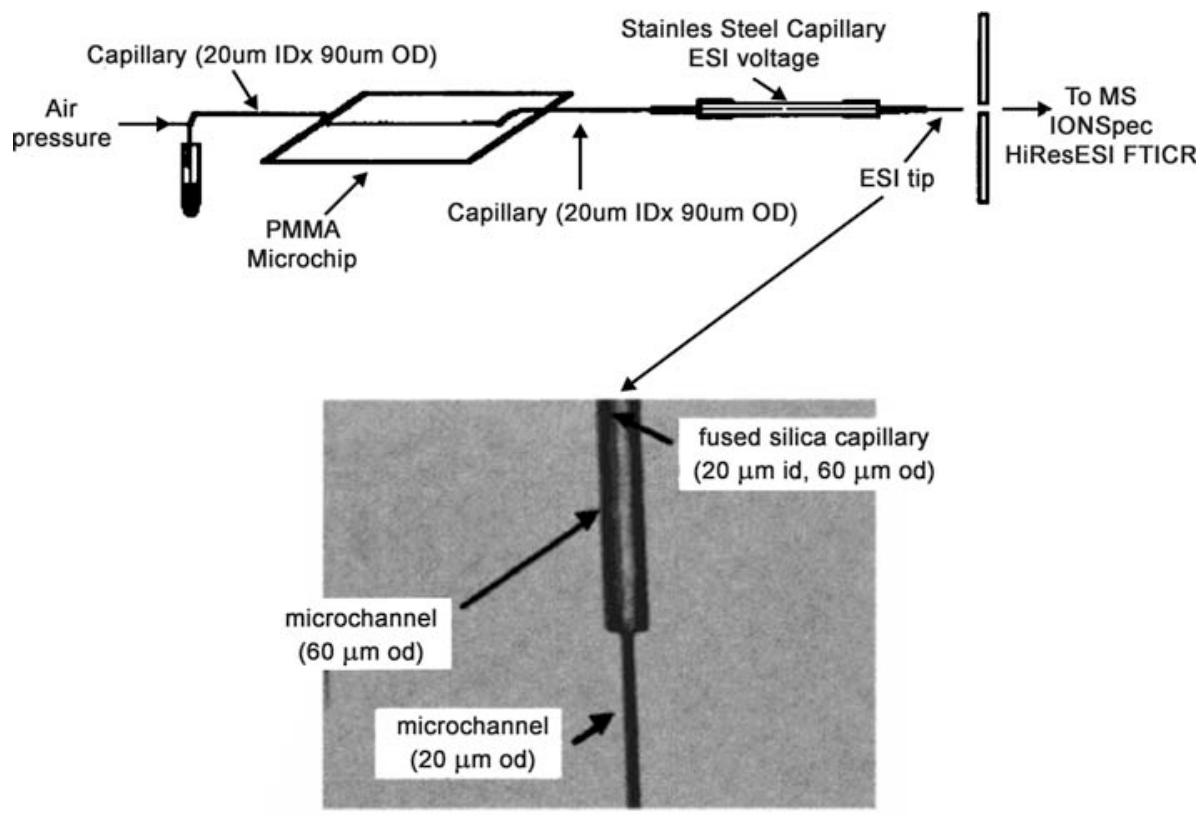

On-Chip Electrospray, Figure 4 On-chip ESI-MS device employing a fused silica transfer capillary. The electrical contact was established through a stainless steel capillary connected to the silica capillary using a poly(tetrafluoroethylene) sleeve. Another fused silica capillary connected $t$ the other end of the stainless steel capillary was used as the electrospray emitter, as shown by the enlargement in the bottom image. Reprinted with permission from [16] 


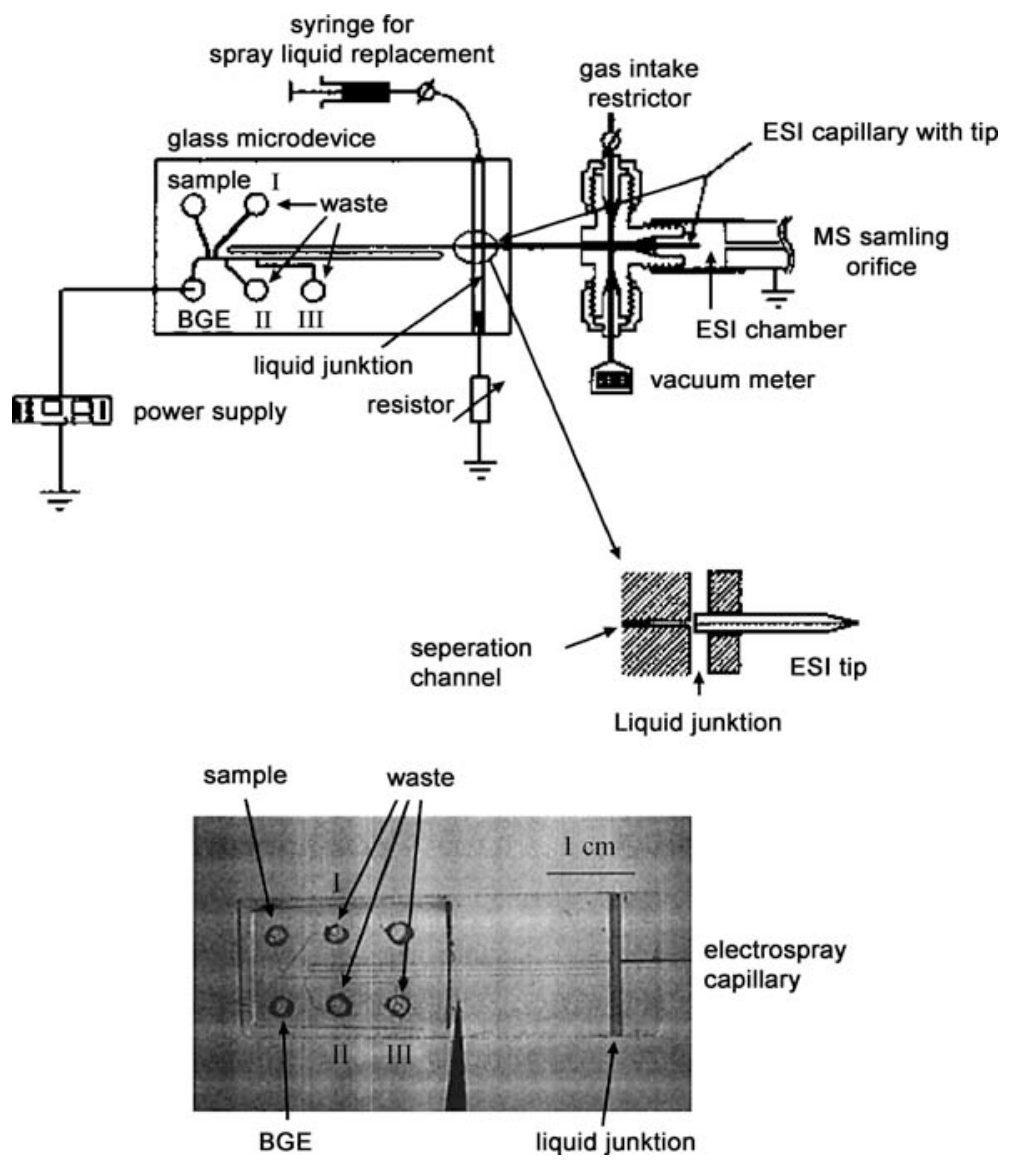

On-Chip Electrospray, Figure 5 Schematic diagram (top) and image (bottom) of the ESI-MS device involving a transfer capillary and a subatmospheric liquid junction. Reprinted with permission from [17]

functioned as the electrospray orifice from which a Taylor cone was observed, thus avoiding the necessity of a dedicated electrospray tip. The coning and spraying is still possible as the electric field intensity at the exposed liquid surface was sufficiently high, above the critical value estimate given by Eq. (2). The blunt edge functioning as the electrospray orifice was formed by either breaking off the scored chip or by cutting the chip using a dicing saw. A similar but parallel multichannel electrospray microchip (Fig. 3) for increased throughput was also developed [12].

Whilst the blunt edge configuration is simple to fabricate, there were several challenges associated with such a design. The low currents associated with electrospraying are insufficient to generate electroosmotic flow (EOF) and hence required the flow to be pressure-driven. The absence of flow regulation however resulted in meniscus ballooning at the orifice which disrupted the electrospray stability and led to the formation of a dead volume; this dead volume introduces hydrodynamic dispersion and hence band broadening of the sample, reducing the efficacy of the separation. Even with application of ancillary electric potentials to drive the flow electroosmotically, the low EOF flow rate made regulation difficult. The electrical connection to the electrospray tip, established by insertion of a metal wire into the sample reservoir, also introduced flow perturbations which aggravated the problem of the lack of flow regulation.

In addition, the ballooning problem was intensified by the hydrophilic glass substrate. This was overcome by surface modification or applying a hydrophobic coating around the edges of the exit end. Nevertheless, this was only a partial remedy as it was possible that the corona discharge affected the chemical stability of the coating [13]. Alternatively, it is possible to bond a porous poly(tetrafluoroethylene) or PTFE membrane at the edge of the microchannel exit acting as the electrospray orifice. The hydrophobic membrane acts to constrain lateral dispersion of the liquid emanating from the orifice. The hydrophobic surface of such a membrane being consis- 
tently hydrophobic and hence the hydrophobicity can be easily controlled [14].

The ballooning problem was also addressed by attaching a pneumatic nebulizer and introducing an ancillary liquid (e. g., acetonitrile, methanol or 2-propanol) sheath flow downstream prior to the electrospray exit [15]. This was carried out by coupling an integrated nebulizer to a subatmospheric liquid junction. The role of the nebulizer gas (e. g., nitrogen) is four-fold, directly removing the ballooning meniscus and hence minimizing the dead volume, controlling the flow rate of the ancillary sheath flow, aiding in the evaporation of the electrosprayed drops, and, providing cooling to the sheath flow at high currents. The role of the sheath flow is two-fold, providing an electrical connection and reducing the ballooning effect and hence dead volume by constraining the flow of the sample. Sheath flow interfaces also enjoy the advantage of wide flexibility in analyte solution selection and enhanced reliability. However, a major drawback of sheath flows is the dilution of the sample and the introduction of background noise from the ancillary flow, both of which compromise the detection sensitivity. The position at which the nebulizer channel merges with the separation channel is however crucial. If this is too far from the exit, the gas pressure results in a back flow whereas non-merger of the channels will result in the failure of the gas flow to disperse the liquid at the exit [15].

Subsequent developments in the on-chip electrospray then involved using either a transfer capillary or a microfabricated emitter tip as a dedicated electrospray orifice in order to more efficiently address these problems.

\section{Capillary Transfer Attachment}

A transfer capillary to which a high voltage is applied can be attached to the edge of a microchannel to act as the electrospray interface. This allows the orifice to be smaller in dimension than that of the blunt edge, thus increasing the stability and hence the performance of the electrospray. In addition, the use of transfer capillaries, the majority constructed from quartz or fused silica, simplifies the construction of the device. Since the capillary is external to the microchannel and chip device, the ESI can be optimized externally. The transfer capillary can also be attached to different chip devices, extending its lifetime and eliminating contamination [13]. An example of the transfer capillary is shown in Fig. 4 in which a long $20 \mathrm{~cm}$ fused silica capillary affixed tightly with epoxy to one end to the microchannel was connected to a stainless steel capillary at its free end with a PTFE tube, which in turn, was connected using the PTFE tubing to an electrospray tip pulled from a fused silica capillary [16].

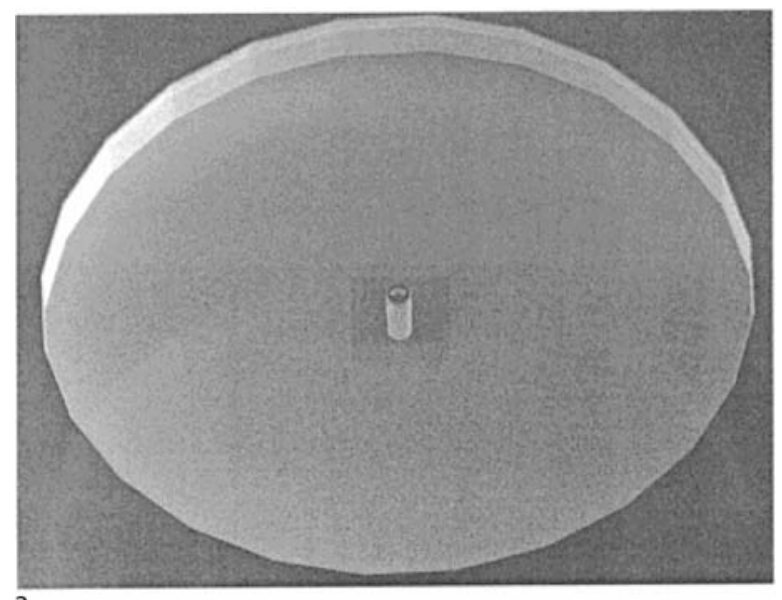

a

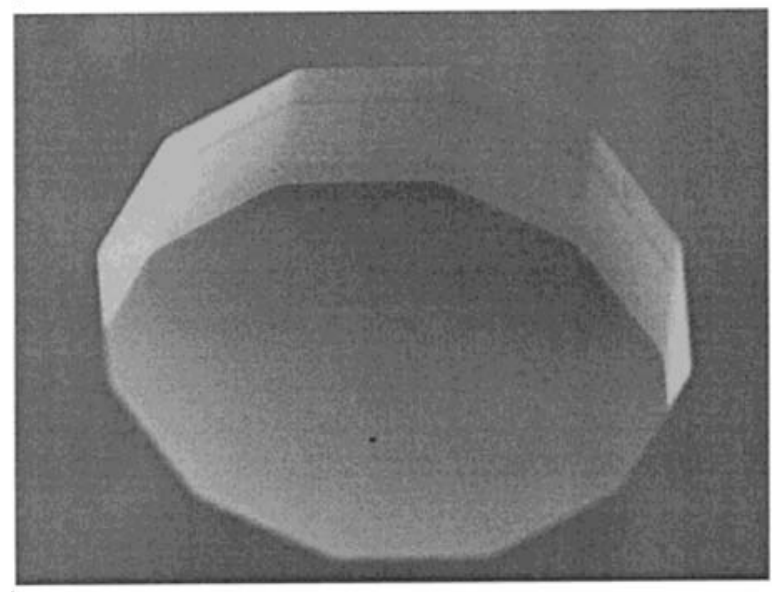
b

On-Chip Electrospray, Figure 6 Scanning electron microscopy images of an out-of-plane needle electrospray emitter tip fabricated at the bottom side of a liquid chamber etched into a silicon wafer. The view from the bottom is depicted in image (a) showing the $10 \mu \mathrm{m}$ diameter needle protruding from the underside of the substrate. The view from the top of the substrate is depicted in image (b) showing the $600 \mu \mathrm{m}$ liquid chamber. Reprinted with permission from [9]

The capillary attachment setup is, however, difficult to control and does not produce good reproducibility. To circumvent these issues, the transfer capillary can also incorporate the sheath flow and liquid junction components discussed above (Fig. 5) to provide the electrical connection, reduce the dead volume as well as to act as a buffer zone such that the separation is not affected by the nebulizer gas flow. Transfer capillaries however require time-consuming alignment and end-to-end butting; poor alignment reintroduces more dead volume instead, resulting in a reduction in the detection sensitivity and separation efficiency. Another deficiency associated with transfer capillary devices is the clogging of the capillary due to contaminants. 


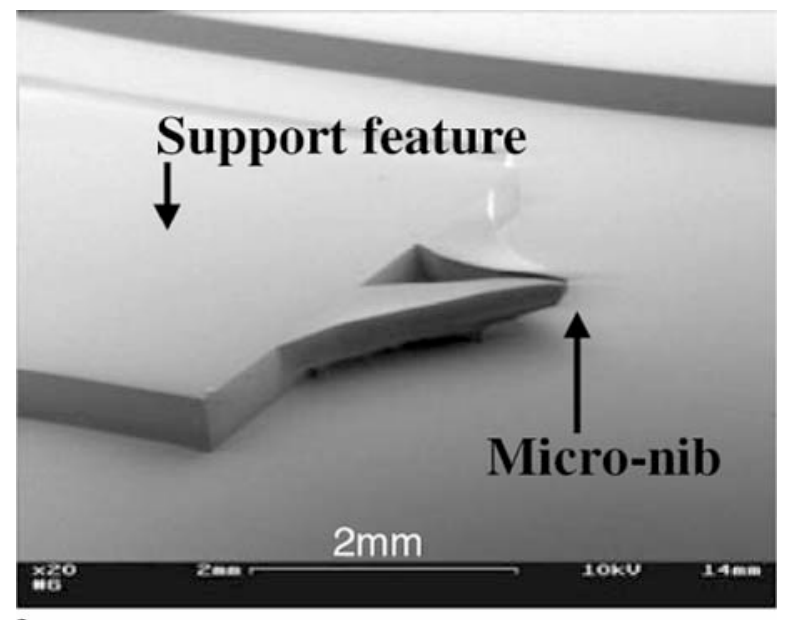

a
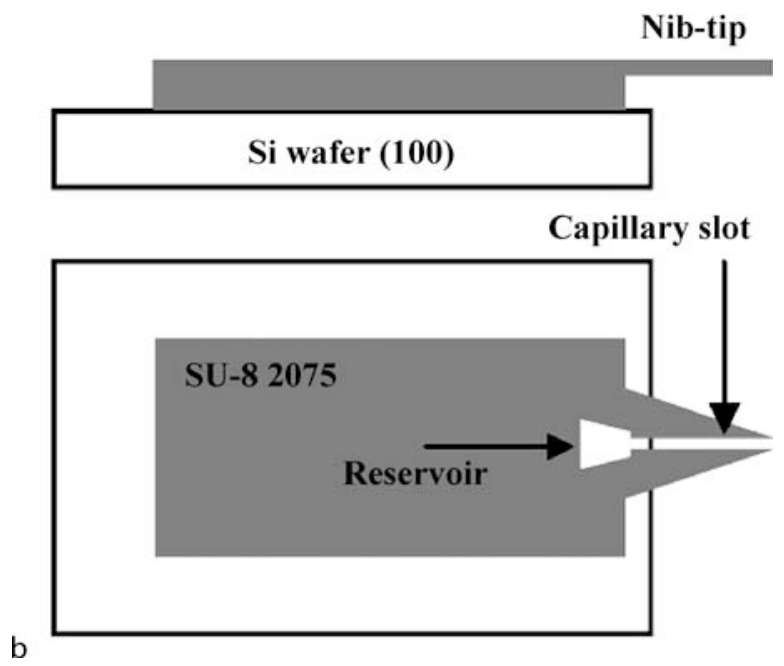

On-Chip Electrospray, Figure 7 Scanning electron microscopy image (a) and schematic plan and side views (b) of the nib-like in-plane electrospray emitter tip fabricated in SU-8 epoxy using double exposure lithography [21]. Reprinted with permission from [21]

Sheathless interfaces have also been developed to eliminate the dilution effect. To achieve a stable electrospray in the absence of nebulizer flow, the capillary tip is often tapered to provide a reduced orifice size. Electrical connection is provided either by applying a conductive coating (e.g., gold, carbon black or a conductive polymer) to the capillary tip, insertion of a wire electrode through a small opening drilled in the capillary wall or with an additional stainless steel capillary connected via a sleeve tubing shown in Fig. 4 [16]. The conductive layer coating is susceptible to damage due to corona discharge and deterioration due to friction. Moreover, these connections suffer the disadvantage of bubble generation due to electrolytic reactions at the electrode, which could disrupt the spraying and suppress the output signal. To avoid this, a Nafion ${ }^{\circledR}$ housing design was employed in a novel sheathless interface device which provides an escape route to relieve the flow of undesirable electrolytic products [18]. Nafion ${ }^{\circledR}$ is a conducting polymer which acts as a chargeselective salt bridge, thus permitting current penetration through the Nafion ${ }^{\circledR}$ membrane, which in itself has negligible polarization due to the small operating current. The external housing of the electrodes thus permits any gas bubbles to escape through the vent. In this same work, an efficient electroosmotic pump is also integrated into the device using silica monoliths, thus enhancing the stability of the electrospray.

\section{Emitter Tips}

Direct electrospraying via emitter tips, also known as nanoelectrospray or nanospray tips, were developed almost as a parallel technique to the transfer capillary interface in an attempt to address the problems associated with direct electrospraying using a blunt edge. The use of emitter tips has the advantage of parallelization in which multiple arrays of emitter tips (over 100 in number) can be fabricated very cheaply and attached onto a microchip, which makes them amenable to high throughput delivery [19]. In general, the development of such tips has taken two routes, namely, out-of-plane tips and in-plane tips. Early work on these emitter tips were almost always out-of-plane, in which the main flow direction and hence emitter is in a direction perpendicular to the device substrate. The first emitter tips comprised of a needle tip, about $50 \mu \mathrm{m}$ at the large end and $5 \mu \mathrm{m}$ at the emitting end, blunt cut to about $2 \mathrm{~cm}$ in length and glued on to the underside of the substrate at a drilled opening where it meets the bottom of the microchannel [20]. The next generation devices consisted of an integrated nozzle fabricated at the bottom of a liquid chamber, as shown in Fig. 6 [9]. Electrical connection was obtained through the use of a conductive coating or a conducting emitter. In another design, an array of $30 \mu \mathrm{m}$ holes was machined into the substrate by laser ablation to function as emitters; the inherent taper of low demagnification factor laser etching processes naturally gives rise to conical orifices [19]. The surfaces of these holes were treated to increase the hydrophobicity.

In-plane emitter tips in which the tips are embedded at the end of the microchannel at the edge of the chip devices have been heralded mainly by recent advances in microfabrication technology by which low cost disposable chips are now close to reality. The smaller dimensions of these emitter tip devices were observed to produce more stable electrosprays over longer times. These emitter tip devices consist of an open capillary slot, connected at one end of 
the chip to a reservoir, which tapers in diameter at the emitting end to form a nib-like structure resembling that of a calligraphic pen, as illustrated in Fig. 7 [21]. This tapered tip is intentionally designed to minimize liquid spreading and to reduce the characteristic orifice length scale and hence the critical voltage required, as stipulated by Eq. (2). In this design, a platinum wire is inserted into the nib to establish the electrical connectivity and sample delivery was achieved using capillary infusion. A drawback of this design, however, is that a large reservoir is required due to evaporation from the open channel. A starshaped chip device containing eight arms, each with a similar open channel and tip for sequential analysis via rotation of the chip has been built [22]. Other tip designs, e. g., groove structures, triangular tips, point tips and enclosed SU-8 tips have also been proposed. To reduce contamination, the insertion of a triangular parylene sheet between a bottom substrate and a top substrate incorporating the channel and tip has been suggested to confine the liquid at the outlet.

\section{Future Directions for Research}

Although a commercially on-chip device for ESI-MS is now available, there still remains several design problems related to enhancing electrospray stability, ionization efficiency, detection sensitivity, separation efficiency, speed, accuracy and spectra quality that need to be addressed. In addition, efforts need to be concentrated to minimize and eliminate sample loss and cross-contamination. Despite significant progress, there is still some way to go before a robust, optimized, cost-effective, high-throughput microdevice providing flexibility for coupling to different MS configurations can be realized. Whilst the high duty cycle of MALDI-MS enables large number of samples deposited on a single target plate, high throughput can only be achieved on chip based ESI-MS devices through the use of multiple channels or emitter tip arrays (see previous section), which themselves are limited by the allotted chip substrate area. The use of high voltage DC electric fields is also a critical safety issue for portable field deployment of miniaturized consumer devices. A possibility around this is to explore the feasibility of employing high frequency AC electric fields [4, 5].

Perhaps the main challenge, however, is the sequential or simultaneous handling of multiple streams (multiplexing) and the integration of multistep processes to allow analyte separation and detection onto a single microchip device [23]. Miniaturization and integration of the mass spectrometer onto the microchip is one goal; some advances have been achieved in scaling down ion trap and quadrupole mass analyzers although the complex- ity of the tandem mass spectrometer poses a significant challenge for its reduction to a chip-based system. The other goal is upstream miniaturization and complete integration of various other processes such as flow-through sampling, and, sample preparation and enrichment. Such a total analysis microsystem would require complete compatibility and connectivity between the various microfluidic components.

\section{Cross References}

- Interfacial Electrokinetic Flow

- Lab-on-Chip Devices for Chemical Analysis

- Lab-on-Chip Devices for Protein Analysis

- Mass Spectrometry

- Mass Spectrometry on-Chip Devices

\section{References}

1. Fenn JB, Mann M, Meng CK, Wong SF, Whitehouse CM (1989) Electrospray ionization for mass spectrometry of large biomolecules. Science 246:64-71

2. Gaskell SJ (1997) Electrospray: Principles and practice. J Mass Spectrom 32:677-688

3. Grace JM, Marijnissen JCM (1994) A review of liquid atomization by electrical means. J Aerosol Sci 25:1005-1019

4. Yeo LY, Lastochkin D, Wang S-C, Chang H-C (2004) A new ac electrospray mechanism by Maxwell-Wagner polarization and capillary resonance. Phys Rev Lett 92:133902

5. Yeo LY, Gagnon Z, Chang H-C (2005) AC electrospray biomaterials synthesis. Biomaterials 26:6122-6128

6. Yeo LY, Chang H-C (2006) High frequency AC electrosprays: Mechanisms and applications. Advances in Fluid Mechanics VI, Skiathos, Greece. WIT Press, Southampton

7. Stone HA, Lister JR, Brenner MP (1999) Drops with conical ends in electric and magnetic fields. Proc R Soc Lond A 455:329-347

8. Thomson BA, Iribarne JV (1979) Field induced ion evaporation from liquid surfaces at atmospheric pressure. J Chem Phys 71:4451-4463

9. Schultz GA, Corso TN, Prosse SJ, Zhang S (2000) A fully integrated monolithic microchip electrospray device for mass spectrometry. Anal Chem 72:4058-4063

10. Licklider L, Wang X-Q, Desai A, Tai Y-C, Lee TD (2000) A micromachined chip-based electrospray source for mass spectrometry. Anal Chem 72:367-375

11. Ramsey RS, Ramsey JM (1997) Generating electrospray from microchip devices using electroosmotic pumping. Anal Chem 69:1174-1178

12. Xue Q, Foret F, Dunayevskiy YM, Zavracky PM, McGruer NE, Karger BL (1997) Multichannel microchip electrospray mass spectrometry. Anal Chem 69:426-430

13. Sung W-C, Makamba H, Chen S-H (2005) Chip-based microfluidic devices coupled with electrospray ionization-mass spectrometry. Electrophoresis 26:1783-1791

14. Wang Y-X, Cooper JW, Lee CS, DeVoe DL (2004) Efficient electrospray ionization from polymer microchannels using integrated hydrophobic membranes. Lab Chip 4:363-367 
15. Zhang B, Liu H, Karger BL, Foret F (1999) Microfabricated devices for capillary electrophoresis-electrospray mass spectrometry. Anal Chem 71:3258-3264

16. Meng Z, Qi S, Soper SA, Limbach PA (2001) Interfacing a polymer-based micromachined device to a nanoelectrospray ionization Fourier transform ion cyclotron resonance mass spectrometer. Anal Chem 73:1286-1291

17. Zhang B, Foret F, Karger BL (2000) A microdevice with integrated liquid junction for facile peptide and protein analysis by capillary electrophoresis/electrospray mass spectrometry. Anal Chem 72:1015-1022

18. Wang P, Chen Z, Chang H-C (2006) A new electro-osmotic pump based on silica monoliths. Sens Actuat B 113:500-509

19. Tang K, Lin Y, Matson DW, Kim T, Smith RD (2001) Generation of multiple electrosprays using microfabricated emitter arrays for improved mass spectrometric sensitivity. Anal Chem 73:1658-1663

20. Lazar IM, Ramsey RS, Sundberg S, Ramsey JM (1999) Subattomole-sensitivity microchip nanoelectrospray source with time-of-flight mass spectrometry detection. Anal Chem 71:3627-3631

21. Arscott S, Le Gac S, Druon C, Tabourier P, Rolando C (2004) A planar on-chip micro-nib interface for NanoESI-MS microfluidic applications. J Micromech Microeng 14:310-316

22. Yuan C-H, Shiea J (2001) Sequential electrospray analysis using sharp-tip channels fabricated on plastic chip. Anal Chem 73:1080-1083

23. Oleschuk RD, Jed Harrison D (2000) Analytical devices for mass spectrometry. Trends in Anal Chem 19:379-388

\section{On-Chip Free-Flow Magnetophoresis}

Magnetophoresis

\section{On-Chip Laboratory}

Lab-on-a-Chip Devices for Chemical Analysis

\section{On Chip Light Confiners}

- On Chip Waveguides

\section{On Chip Light Guiding}

- On Chip Waveguides

\section{On Chip Light Propagator}

- On Chip Waveguides

\section{On-Chip Micro-Optical Detection}

\author{
HOLGER SCHMIDT \\ University of California, Santa Cruz, CA, USA \\ hschmidt@soe.ucsc.edu
}

\section{Synonyms \\ On-chip detection}

\section{Definition}

On-chip micro-optical detection describes the collection of optical signals created in a micro- or nanofluidic device on the same chip. The wavelengths of the optical signals to be detected are typically in the visible or near-infrared part of the spectrum ranging from 0.4 to $2 \mu \mathrm{m}$. This sets the length scale for the detection elements and results in the use of micro-optical methods for on-chip detection. The detection process is an integral part of a fully integrated Lab-on-a-Chip and has to be optimized in the context of the entire chip. In this chapter, we will survey the most common approaches to on-chip micro-optical detection in the context of the complete integrated microfluidic system.

\section{Overview}

A large number of microfluidic devices with applications in analytical chemistry, biology, and biomedicine rely at least in part on optical methods for sample analysis. At the same time, the overarching goal for building microfluidic systems is to create miniaturized, portable, and selfcontained Labs-on-a-Chip that perform all desired functions in as small a volume as possible. If optical analysis is to be part of the fluidic system, all optical components should be miniaturized as well. This situation is schematically depicted in Fig. 1a that shows the components of a Lab-on-a-Chip with focus on the optical parts. In general terms, light has to be brought in contact with liquid sample material. To this end, the outputs of light and fluid sources are guided via optical waveguides and fluidic channels, respectively, to an interaction region denoted by "I" where the sample analyte creates a characteristic optical signal, typically fluorescence, scattering, or absorption. This optical signal needs to be recorded by the detector, and to this end may pass additional functional optical elements such as filters, beam splitters, or gratings. Fig. $1 \mathrm{~b}$ shows a recent example for an optofluidic chip that incorporates all these elements in a single device, resulting in almost complete integration [1]. In the remainder of this article, we will first give a brief overview of the optical components preceding the detector stage before focusing on proposed and cur- 